\title{
Dynamic visual servo control of a 4-axis joint tool to track image trajectories during machining complex shapes
}

\author{
Jorge Pomares*, Iván Perea, Carlos A. Jara, Gabriel J. García, Fernando Torres \\ Department of Physics, System Engineering and Signal Theory \\ University of Alicante, San Vicente del Raspeig, Spain \\ POBox 99, 03080, Alicante, Spain \\ \{jpomares, ivan.perea, carlos.jara, gggg, Fernando.torres\}@ua.es
}

* Corresponding author

E-mail address: jpomares@ua.es (Jorge Pomares).

Department of Physics, System Engineering and Signal Theory

University of Alicante, San Vicente del Raspeig, Spain

POBox 99, 03080, Alicante, Spain

Tel: (+34) 965903400 - 2032

Fax: (+34) 965909750 


\title{
Dynamic visual servo control of a 4-axis joint tool to track image trajectories during machining complex shapes
}

\begin{abstract}
A large part of the new generation of computer numerical control systems has adopted an architecture based on robotic systems. This architecture improves the implementation of many manufacturing processes in terms of flexibility, efficiency, accuracy and velocity. This paper presents a 4-axis robot tool based on a joint structure whose primary use is to perform complex machining shapes in some non-contact processes. A new dynamic visual controller is proposed in order to control the 4-axis joint structure, where image information is used in the control loop to guide the robot tool in the machining task. In addition, this controller eliminates the chaotic joint behaviour which appears during tracking of the quasi-repetitive trajectories required in machining processes. Moreover, this robot tool can be coupled to a manipulator robot in order to form a multi-robot platform for complex manufacturing tasks. Therefore, the robot tool could perform a machining task using a piece grasped from the workspace by a manipulator robot. This manipulator robot could be guided by using visual information given by the robot tool, thereby obtaining an intelligent multi-robot platform controlled by only one camera.
\end{abstract}

Keywords: visual servoing, tracking image trajectories, direct control, robotics.

\section{Introduction}

The complexity of geometric shapes, the accuracy needed in the finished worked piece and the high speed required for the machining processes are some of the current requirements for the new generation of computer numerical control (CNC) systems. Complex CNC architectures, most of them based on robotic systems $[1][2][3][4]$, have been developed in order to provide more flexibility, efficiency, accuracy, and velocity in the implementation of many manufacturing processes. In this context, robotics technology permits machining via different working methods (e.g. milling, polishing, cutting) thus enabling higher working efficiency. In addition, commercial manufacturing machines are expensive and their utilization in some cases is quite limited in practice due to their low versatility and flexibility. For these reasons, research about the development of reconfigurable CNC manufacturing machines has increased in the last few years [4][5][6][7]. This paper proposes a 4-axis robot tool (RT) based on a joint structure whose main use will be to perform complex machining shapes in some noncontact processes such as laser and water cutting. This RT can be coupled to a robotic system in order to perform complex manufacturing tasks. A new dynamic visual controller is proposed to control the 4-axis joint structure, where image information is used in the control loop to guide the RT in its machining task. 
CNC manufacturing machines are normally controlled without vision. Only few works have dealt with the introduction of vision in $\mathrm{CNC}$ machining processes. In [8], it is presented an approach to improve the performance of CNC machining by utilizing on-line vision-based monitoring. A stereo camera configuration is employed to monitor the milling task, but there is no direct control of the position using the vision system. This same scheme is used before in [9], introducing vision for inspection of the CNC machining task. Thus, vision has been applied to CNC systems only for monitoring or inspection tasks. A computer vision system is applied in [10] to the generation of tool path in a previous phase of the milling task. In this paper, vision is used to directly control the CNC system.

High-speed machining processes have great dynamic requirements on the structure, which are further augmented by the acceleration of different parts of the machine or robotic platform. This may cause some alterations of the tool's position and orientation errors [3][11]. For these reasons, control of the machine's engine can be a difficult task utilizing position, orientation or velocity sensor data. Currently, there are many approaches where a new control system is proposed for machine's axes [3][12][13]. The dynamic visual controller proposed here permits accurate guidance of the RT's end-effector, guaranteeing the correct joint motion. The classic imagebased controllers determine the required camera velocities in order to position the robot with respect a reference object [14]. Using this last approach, the internal robot controller computes the joint torques in order to achieve the previously mentioned camera velocities. However, the approach proposed in this paper to perform the RT guidance is based on direct visual servoing [15]. By means of direct visual servo, the internal control loop of servo motors is removed and the visual servo control is employed to stabilize the robot. Thus, the use of direct control eliminates the delay introduced by the use of an additional controller as it is the robot internal controller. The result is a faster and more accurate control that reacts more quickly to abrupt changes in the trajectories. This proposed visual controller performs high precision visual tracking in order to be applied to CNC machines. Additionally, an important contribution of the proposed controllers is to eliminate the chaotic joint behaviour which appears during tracking of the quasi-repetitive trajectories required in the machining process.

In machining processes such as polishing, milling or cutting, the RT must track repetitive or quasi-repetitive trajectories. As described through the paper, a repetitive path tracked by the RT end-effector can produce a nonperiodic motion in the joint space. This non-periodic joint trajectory presents a chaotic behavior, an undesirable nonlinear effect which can appear during some machining processes [16][17]. This chaotic behavior has embedded a large number of Unstable Periodic Orbits (UPOs). As previously indicated, the proposed visual controller determines appropriate motor references such that both RT's end-effector and joints behave correctly. To do this, a chaos compensator is integrated within the dynamic visual controller to eliminate the chaotic joint behavior and to obtain a quasi-repetitive joint trajectory when the end-effector also tracks a quasi-repetitive trajectory. This last aspect guarantees a smoother joint behavior and increases the safety obtaining predictable trajectories. This property improves the performance and accuracy of CNC machines as part of an intelligent robotic system. 
Different kinematic configurations are studied in order to couple the RT to the end of another manipulator robot in order to perform complex machining tasks. The RT components and their constructive properties have been designed in order to facilitate its installation without any constraints to the manipulator robot functionalities. Therefore, the RT could perform a given machining task using a piece grasped from the workspace by the manipulator robot. The manipulator robot could be guided by visual information given by the RT, thereby obtaining an intelligent multi-robot platform controlled by only one camera.

This paper is organized as follows. Section 2 describes the kinematic and dynamic features of the RT, as well as showing uses for a multi-robot platform. Section 3 explains the visual control of the RT. At the beginning of this section, the dynamics and kinematics notation of the robot model are shown in detail. Section 4 describes the proposed controller used to track trajectories in the image space to solve the chaotic behaviour of the RT. Section 5 verifies the controller using different velocities and the final section elaborates the conclusions reached by this paper and possible future works.

\section{Robot Tool Kinematics and Dynamics}

This section describes the main kinematic and building properties of the RT. The RT is shown in Figure 1, where $1_{1}=12.4 \mathrm{~cm}, l_{2}=15.2 \mathrm{~cm}, l_{3}=9.9 \mathrm{~cm}$. The corresponding mass properties of each link are $\mathrm{m}_{1}=1.75 \mathrm{~kg}, \mathrm{~m}_{2}=0.8$ $\mathrm{kg}, \mathrm{m}_{3}=0.5 \mathrm{~kg}$ respectively. The robot is guided by an eye-in-hand camera system. A Gigabit Ethernet TM6740GEV camera is used, which acquires 200 images every second with a resolution of 640x480 pixels. Four rotational joints are employed $\left(\mathrm{q}_{0}, \mathrm{q}_{1}, \mathrm{q}_{2}, \mathrm{q}_{3}\right)$ for the RT movement. The proposed controllers shown throughout the paper can be easily extended to a great number of joints. However, four degrees of freedom (d.o.f.) have been considered sufficient for a tool to perform the necessary machining and positioning tasks.

The first joint has been designed with an inner space that permits affixing the RT at the end of a manipulator robot (without negatively affecting the functionality of the manipulator robot). The first joint has a rotational movement, $\mathrm{q}_{0}$, independent of the robot manipulator and the other three joints, $\mathrm{q}_{1}, \mathrm{q}_{2}$ and $\mathrm{q}_{3}$, are used to undertake the machining task. The main objective of the proposed design is to create a robust kinematic and dynamic structure with its basis in a known model, guided by direct visual servoing. As it is previously indicated, the RT is designed to be placed and coupled at the end of a manipulator robot. Therefore, the lightness of the RT is an important aspect to be considered in the design. For this reason, strong and lightweight materials (mainly duralumin and high resistance plastics) have been employed in its construction, resulting in a total weight of $5 \mathrm{Kg}$. 


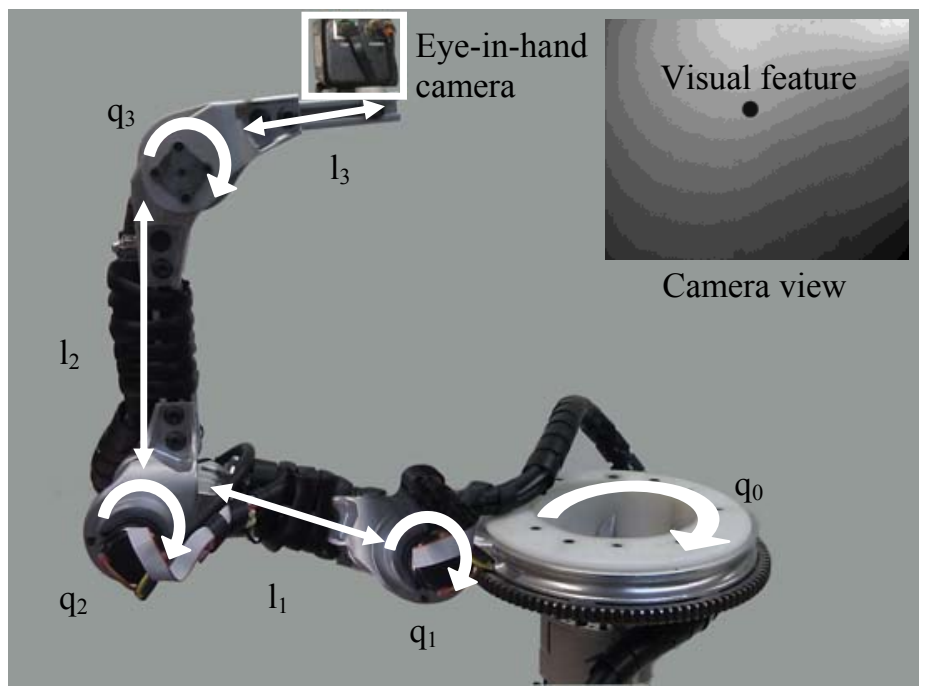

Figure 1. Robot tool

In order to select the actuators, drivers, and power control devices for the RT, the 3D model design and the dynamic simulation of the robot movement were made. From the data calculated in this simulation, four geared motors (one per joint) were considered for the robot actuators. Table 1 shows the features of the selected engine motors of the joints $\mathrm{q}_{0}, \mathrm{q}_{1}, \mathrm{q}_{2}$ and $\mathrm{q}_{3}$, where $\mathrm{M}_{\mathrm{b}}(\mathrm{N} \cdot \mathrm{m})$ is the torque value, $\mathrm{N}_{\mathrm{b}}(\mathrm{rpm})$ is the load speed engine, and $\eta_{\mathrm{m}}$ (\%) is the energy efficiency. Table 2 shows the features of the selected gears, where $R$ is the reduction, $M_{\text {cont }}(\mathrm{N} \cdot \mathrm{m})$ and $\mathrm{M}_{\mathrm{int}}(\mathrm{N} \cdot \mathrm{m})$ are the nominal and maximum torque supported by the device, and $\eta_{\mathrm{r}}(\%)$ is the energy efficiency. The maximum torque of each geared-motor group was computed using $N_{f}=M_{b} \cdot R \cdot \eta_{r} \cdot \eta_{m}$. The results obtained for the torques were $\tau_{0 \mathrm{f}}=25.06 \mathrm{~N} \cdot \mathrm{m}, \tau_{1 \mathrm{f}}=16.03 \mathrm{~N} \cdot \mathrm{m}, \tau_{2 \mathrm{f}}=9.05 \mathrm{~N} \cdot \mathrm{m}$ and $\tau_{3 \mathrm{f}}=6.54 \mathrm{~N} \cdot \mathrm{m}$.

Table 1: Motor parameters of the joints $\mathrm{q}_{0}, \mathrm{q}_{1}, \mathrm{q}_{2}, \mathrm{q}_{3}$

\begin{tabular}{cccc}
\hline Motor & $\mathrm{M}_{\mathrm{b}}(\mathrm{Nm})$ & $\mathrm{N}_{\mathrm{b}}(\mathrm{rpm})$ & $\eta_{\mathrm{m}}(\%)$ \\
\hline Joint $\mathrm{q}_{0}$ & 0.510 & 6930 & 91 \\
Joint $\mathrm{q}_{1}$ & 0.316 & 10000 & 87 \\
Joint $\mathrm{q}_{2}$ & 0.131 & 15900 & 89 \\
Joint $\mathrm{q}_{3}$ & 0.0504 & 14700 & 88 \\
\hline
\end{tabular}

Table 2: Gear parameters of the joints $\mathrm{q}_{0}, \mathrm{q}_{1}, \mathrm{q}_{2}, \mathrm{q}_{3}$

\begin{tabular}{ccccc}
\hline Gear & $\mathrm{R}$ & $\mathrm{M}_{\text {cont }}(\mathrm{Nm})$ & $\mathrm{M}_{\text {int }}(\mathrm{Nm})$ & $\eta_{\mathrm{r}}(\%)$ \\
\hline Joint $\mathrm{q}_{0}$ & 75 & 20 & 27.5 & 72 \\
Joint $\mathrm{q}_{1}$ & 81 & 15 & 22.5 & 72 \\
Joint $\mathrm{q}_{2}$ & 111 & 8 & 12 & 70 \\
Joint $\mathrm{q}_{3}$ & 246 & 6 & 7.5 & 60 \\
\hline
\end{tabular}

The RT is designed to be installed at the end of a manipulator robot. This permits machining a piece grasped by the manipulator robot, as well as allowing the manipulator guidance using image information extracted by the RT. In a previous work [18], the authors have described techniques for guiding two robots (one coupled at the end of another) by using image information obtained by the coupled robot. However, the RT has an additional d.o.f. with regard to the coupled robot employed in [18] and the motor properties are not the same. Furthermore, the 
controller presented in this paper has several contributions compared with the previous ones with the aim to be applied to machining tasks. The previous controller only permits positioning tasks (it does not allow the tracking of image trajectories). The controller presented here is the first dynamic visual servo controller which integrates the correction of joint chaos in the tracking of image trajectories. The controller implements a direct visual servo control that integrates manipulability in the redundancy resolution. Furthermore, in order to guarantee a correct behavior in practice, a method to adjust the chaos controller is proposed and validated using the experimental results.

\section{Visual control of the 4-axis Robot Tool}

This section shows the controller proposed to visually guide the RT during its machining task. With consideration to the general case of a n-link rigid robot mechanism, the dynamics can be written as:

$$
\mathbf{M}(\mathbf{q}) \ddot{\mathbf{q}}+\mathbf{C}(\mathbf{q}, \dot{\mathbf{q}})+\mathbf{g}(\mathbf{q})=\boldsymbol{\tau}
$$

where $\mathbf{q} \in \mathfrak{R}^{\mathrm{nx} 1}$ is the set of joint positions, $\dot{\mathbf{q}} \in \mathfrak{R}^{\mathrm{nx} 1}$ represents the joint velocities vector and $\ddot{\mathbf{q}} \in \mathfrak{R}^{\mathrm{nx} 1}$ are the joint accelerations. Furthermore, $\mathbf{M}(\mathbf{q}) \in \mathfrak{R}^{\mathrm{nxn}}$ is the symmetric and positive definite inertia matrix, $\mathbf{C}(\mathbf{q}, \dot{\mathbf{q}}) \in \mathfrak{R}^{\mathrm{nx} 1}$ is the vector of centripetal and Coriolis torques and $\mathbf{g}(\mathbf{q})$ is the gravity vector. Finally, $\boldsymbol{\tau} \in \mathfrak{R}^{\mathrm{nx}}$ is the effective joint torques vector.

As previously indicated, the RT is guided by visual information. This visual information is a vector of $\mathrm{k}$

image point features $\mathbf{s}=\left[f_{1 \mathrm{x}}, f_{1 \mathrm{y}}, f_{2 \mathrm{x}}, f_{2 \mathrm{y}}, \ldots, f_{\mathrm{kx}}, f_{\mathrm{ky}}\right]^{\mathrm{T}} \in \mathfrak{R}^{2 \mathrm{k}}$, which are extracted from a captured image. Denoting $\dot{\mathbf{s}}$ as the time derivative of the image features, the following relation is established by the interaction matrix $\mathbf{L}_{\mathrm{s}}$ [14]:

$$
\dot{\mathbf{s}}=\mathbf{L}_{\mathrm{s}}(\mathbf{r}) \dot{\mathbf{r}}_{\mathrm{c}}
$$

where $\mathbf{r}_{\mathrm{c}}$ denotes the pose of the camera located at the RT end-effector and $\dot{\mathbf{r}}_{\mathrm{c}}$ is the corresponding velocity. Although, the interaction matrix depends on the intrinsic camera parameters and the depth of the visual features, several works have demonstrated that IBVS systems converge to the desired position when these parameters are not perfectly estimated [19]. In this paper, it will be assumed that the intrinsic and extrinsic camera parameters have been obtained using an off-line classical calibration process [20]. The robot-end is performing a task in an $\mathrm{m}$ dimensional space, therefore, the end-effector pose $\mathbf{r} \in \mathfrak{R}^{\mathrm{m}}$. In order to obtain the end-effector velocity, $\dot{\mathbf{r}}$, through the camera velocity, $\dot{\mathbf{r}}_{\mathrm{c}}$, a twist matrix transformation is needed: 


$$
\dot{\mathbf{r}}=\left[\begin{array}{cc}
{ }_{\mathrm{c}}^{\mathrm{e}} \mathbf{R} & { }^{\mathrm{e}} \mathbf{P}_{\mathrm{cORG}} \times{ }_{\mathrm{c}}^{\mathrm{e}} \mathbf{R} \\
0 & { }_{\mathrm{c}}^{\mathrm{e}} \mathbf{R}
\end{array}\right] \dot{\mathbf{r}}_{\mathrm{c}}={ }_{\mathrm{c}}^{\mathrm{e}} \mathbf{T} \dot{\mathbf{r}}_{\mathrm{c}}
$$

where ${ }_{\mathrm{c}}^{\mathrm{e}} \mathbf{R}$ represents the rotation of the camera frame with respect to the end-effector frame and ${ }^{\mathrm{e}} \mathbf{P}_{\mathrm{cORG}}$ is the translation between camera and end-effector frames.

The end-effector velocity is related to the joint-space velocity $\dot{\mathbf{q}}$ by the robot Jacobian:

$$
\dot{\mathbf{r}}=\mathbf{J}_{\mathrm{r}}(\mathbf{q}) \dot{\mathbf{q}}
$$

From (2), (3) and (4) the following relation can be established:

$$
\dot{\mathbf{s}}=\mathbf{L}_{\mathrm{s}}(\mathbf{r})_{\mathrm{c}}^{\mathrm{e}} \mathbf{T}^{-1} \mathbf{J}_{\mathrm{r}}(\mathbf{q}) \dot{\mathbf{q}}=\mathbf{J}(\mathbf{q}) \dot{\mathbf{q}}
$$

where $\mathbf{J}=\mathbf{J}(\mathbf{q}) \in \mathfrak{R}^{2 \mathrm{k} \times \mathrm{n}}$ is the Jacobian matrix mapping from joint space to image space. The image acceleration $\ddot{\mathbf{s}}$ can be obtained by differentiating (5) with respect to the time:

$$
\ddot{\mathbf{s}}=\mathbf{J} \ddot{\mathbf{q}}+\dot{\mathbf{J}} \dot{\mathbf{q}}
$$

This last equation relates the joint accelerations and the second derivatives of the image features vector. Using a dynamic model-based compensation approach, the joint torques can be obtained using the following equation:

$$
\boldsymbol{\tau}=\mathbf{M}(\mathbf{q}) \ddot{\mathbf{q}}_{\mathrm{r}}+\mathbf{C}(\mathbf{q}, \dot{\mathbf{q}})+\mathbf{g}(\mathbf{q})
$$

where $\ddot{\mathbf{q}}_{\mathrm{r}}$ represents a resolved acceleration in terms of joint variables. The goal of the proposed controller is to obtain the required torques, thereby ensuring correct tracking of the desired image trajectory during the machining task. The control input $\ddot{\mathbf{q}}_{\mathrm{r}}$ can be obtained from (6) as:

$$
\ddot{\mathbf{q}}_{\mathrm{r}}=\mathbf{J}^{+}\left(\ddot{\mathbf{s}}_{\mathrm{r}}-\dot{\mathbf{J}} \dot{\mathbf{q}}\right)
$$

where $\ddot{\mathbf{s}}_{\mathrm{r}}$ is the resolved acceleration in terms of extracted image features, and $\mathbf{J}^{+}$denotes the Moore-Penrose pseudoinverse of $\mathbf{J}$. Two different tasks are defined as follows:

- Primary task (Equation (8)): performs the tracking of a desired image trajectory.

- Secondary task: expressed as a cost function to be minimized, with the prerequisite that the primary task is satisfied. The secondary task is employed to avoid robot singularities. Yoshikawa [21] proposed the term manipulability, which measures the distance of any redundant configuration from singular ones. The manipulability, $\omega_{\mathrm{r}}$, can be obtained by:

$$
\omega_{\mathrm{r}}=\sqrt{\operatorname{det}\left(\mathbf{J}_{\mathrm{r}}^{\mathrm{T}}(\mathbf{q}) \mathbf{J}_{\mathrm{r}}(\mathbf{q})\right)}
$$

Therefore, the new control input for the RT's accelerations using the secondary and primary tasks is: 


$$
\ddot{\mathbf{q}}_{\mathrm{r}}=\mathbf{J}^{+}\left(\ddot{\mathbf{s}}_{\mathrm{r}}-\dot{\mathbf{J}} \dot{\mathbf{q}}\right)+\alpha\left(\mathbf{I}-\mathbf{J}^{+} \mathbf{J}\right) \cdot \nabla \omega_{\mathrm{r}}
$$

where the parameter $\alpha$ is a positive gain and $\mathbf{I}-\mathbf{J}^{+} \mathbf{J}$ projects $\nabla \omega_{\mathrm{r}}$ onto the null space of the Jacobian $\mathbf{J} . \nabla \omega_{\mathrm{r}}$ is interpreted as a desired behavior that is only effective in the null space.

The resolved acceleration in the image space is calculated by the following equation:

$$
\ddot{\mathbf{s}}_{\mathrm{r}}=\ddot{\mathbf{s}}_{\mathrm{d}}+\mathbf{K}_{\mathrm{D}}\left(\dot{\mathbf{s}}_{\mathrm{d}}-\dot{\mathbf{s}}\right)+\mathbf{K}_{\mathrm{P}}\left(\mathbf{s}_{\mathrm{d}}-\mathbf{s}\right)=\ddot{\mathbf{s}}_{\mathrm{d}}+\mathbf{K}_{\mathrm{D}} \dot{\mathbf{e}}_{\mathrm{s}}+\mathbf{K}_{\mathrm{P}} \mathbf{e}_{\mathrm{s}}
$$

where $\ddot{\mathbf{s}}_{\mathrm{d}}, \dot{\mathbf{s}}_{\mathrm{d}}$ and $\mathbf{s}_{\mathrm{d}}$ are the desired image space accelerations, velocities and positions, respectively. The set of desired visual features $\mathbf{s}_{\mathrm{d}}$ depends on the time, $\mathbf{s}_{\mathrm{d}}(\mathrm{t})$. For the sake of clarity, these desired features are referenced as $\mathbf{s}_{\mathrm{d}}$ in the rest of the paper. Therefore, the image trajectory to be tracked is a time-dependent function. $\mathbf{K}_{\mathrm{P}}$ and $\mathbf{K}_{\mathrm{D}}$ are the PD gain matrices. Also, $\mathbf{e}_{\mathrm{s}}$, is the image error and $\dot{\mathbf{e}}_{\mathrm{s}}$ is the time derivative of the image error.

From (7) and (10):

$$
\boldsymbol{\tau}=\mathbf{M}(\mathbf{q}) \mathbf{J}^{+}\left(\ddot{\mathbf{s}}_{\mathrm{r}}-\dot{\mathbf{J}} \dot{\mathbf{q}}\right)+\alpha \mathbf{M}(\mathbf{q})\left(\mathbf{I}-\mathbf{J}^{+} \mathbf{J}\right) \cdot \nabla \omega_{\mathrm{r}}+\mathbf{C}(\mathbf{q}, \dot{\mathbf{q}})+\mathbf{g}(\mathbf{q})
$$

The closed loop behavior can be obtained from (1) and (12):

$$
\mathbf{M}(\mathbf{q}) \ddot{\mathbf{q}}=\mathbf{M}(\mathbf{q}) \mathbf{J}^{+}\left(\ddot{\mathbf{s}}_{\mathrm{r}}-\dot{\mathbf{J}} \dot{\mathbf{q}}\right)+\alpha \mathbf{M}(\mathbf{q})\left(\mathbf{I}-\mathbf{J}^{+} \mathbf{J}\right) \cdot \nabla \omega_{\mathrm{r}}
$$

In order to guarantee the applicability of the proposed visual servoing system, a repetitive (or quasi-repetitive) image trajectory tracked by the RT end-effector must produce a periodic (or quasi-periodic) joint motion. For example, accurate tracking of a spiral image trajectory of a laser milling process as the shown in Figure 2.a, could be obtained with a quasi-periodic joint motion. However, Sections 4 and 5 describe different experiments where a nonperiodic joint motion is obtained during tracking of a periodic or spiral image trajectory. A chaotic joint behavior is observed using the proposed dynamic visual control system during tracking of these trajectories. A Delayed Feedback Control (DFC) is integrated in the proposed visual servo controller in order to avoid chaotic behavior in the joint space during tracking of these image trajectories. The DFC method, proposed by Pyragas [22], has proven adequate for controlling chaotic systems. The DFC consists of a continuous feedback from the difference between the current state and the delayed state of the system. To implement the DFC method, the joint velocity variables are considered as the system state variables. Therefore, the new resolved acceleration with the DFC term leads to:

$$
\ddot{\mathbf{q}}_{\mathrm{r}}=\mathbf{J}^{+}\left(\ddot{\mathbf{s}}_{\mathrm{r}}-\dot{\mathbf{J}} \dot{\mathbf{q}}\right)+\alpha\left(\mathbf{I}-\mathbf{J}^{+} \mathbf{J}\right) \cdot \nabla \omega_{\mathrm{r}}+\mathrm{k}(\dot{\mathbf{q}}(\mathrm{t}-\varepsilon)-\dot{\mathbf{q}}(\mathrm{t}))
$$

where $\mathrm{k}$ is a constant gain which requires appropriate adjustments in order to stabilize the target UPO. $\varepsilon$ is the feedback time delay, and it is chosen so as to be equal to the period of the target UPO. This value is assumed to be known a priori. 


\section{Adaptive controller for complex shapes: solving chaotic behaviour in the RT}

The previous section proposed a method for dynamic guidance of the RT using visual information, and a DFC approach was included in the controller in order to avoid chaotic behavior during tracking. This section builds on the previous and describes an adaptive method for adjusting the gain value of the DFC part of the controller during tracking.

In order to illustrate the chaotic behavior of the RT and the necessity for an adaptive method for adjusting the controller, this section proposes an initial experiment. This experiment consists of a laser milling process which requires tracking a spiral trajectory of an image point (in order to perform this experiment a laser head is located at the RT-end). This task does not require the use of $\mathrm{q}_{0}$ : only 3 d.o.f. are required to perform the machining task. Since the laser is mounted on the RT-end, the laser speed is controlled via command of the RT joint torques. The milling process is performed in a horizontal plane so that the joints $\mathrm{q}_{1}, \mathrm{q}_{2}$ and $\mathrm{q}_{3}$ should not compensate the gravity force. The milling task is performed over a piece located in a parallel plane with respect to the camera and the laser. The depth of the visual feature is constant $(0.5 \mathrm{~m})$, and is computed previously using a classical calibration process [20].

The trajectory to be tracked by the RT in the image space is defined by:

$$
\mathbf{s}_{\mathrm{d}}=\left[\begin{array}{l}
f_{\mathrm{xd}} \\
f_{\mathrm{yd}}
\end{array}\right]=\left[\begin{array}{l}
305+(160-0.25 \mathrm{t}) \cos (\omega \mathrm{t}+\pi / 4)+8 \sin (\omega 5 \mathrm{t}) \\
300+(110-0.25 \mathrm{t}) \sin (\omega \mathrm{t}+\pi / 4)+6 \sin (\omega 5 \mathrm{t})
\end{array}\right] \mathrm{px}
$$

where the tracking velocity is determined by $\omega$. The image trajectory defined by the equation (15) is illustrated in Figure 2.a, and this trajectory is almost repetitive. Therefore, in the joint space a similar behavior is required, i.e., the joint configuration of the RT must be a quasi-repetitive trajectory. In the following paragraphs, the controller's performance without chaos control is described not only in regards to the image space but also in terms of the joint space. To evaluate the tracking system in a given machining process, an experiment was conducted where the values of the proportional and derivative matrices were: $\mathbf{K}_{\mathrm{P}}=\operatorname{diag}(10,10)$ and $\mathbf{K}_{\mathrm{D}}=\operatorname{diag}(50,50)$. In this experiment, a middle tracking velocity is used $(\omega=0.5 \mathrm{rad} / \mathrm{s})$, and $\mathrm{k}=0$. The obtained image trajectory is represented in Figure 3.a. Figure 3.b describes the error in the image space at each iteration during the tracking $\left(\mathbf{s}_{\mathrm{d}}-\mathbf{s}\right)$. The conclusion drawn from these last figures is that the tracking was correctly carried out in the image space.

The phase portraits are represented in Figure 4 in order to evaluate the joint behavior during the tracking of the image trajectory defined by the Equation (15). The Figure 4 indicates the presence of non-repetitive and chaotic behavior in the joint space. The largest Lyapunov exponent diagram (represented in Figure 5) also indicates the presence of chaotic behavior in the joint space. 


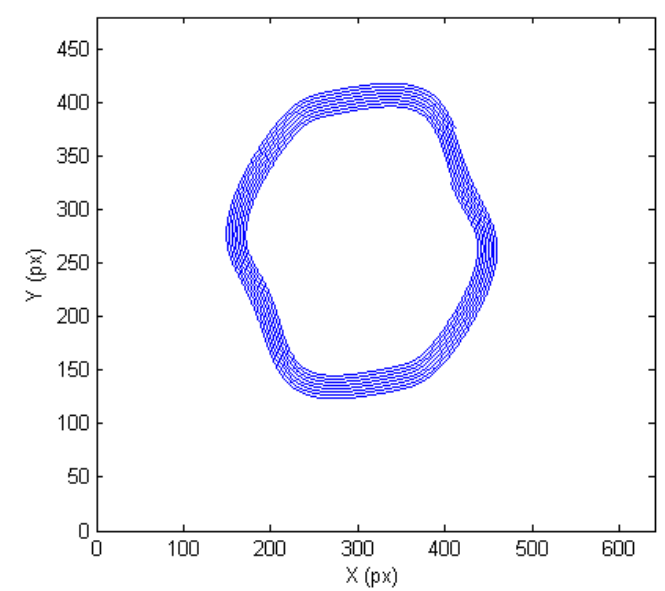

a)

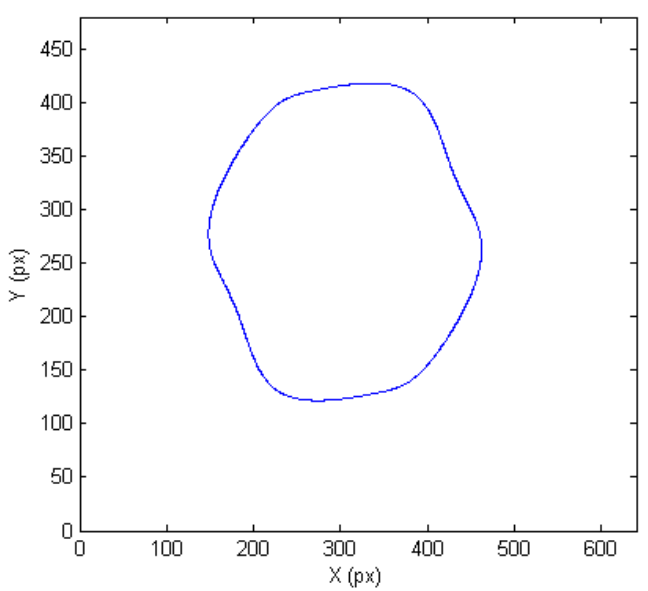

b)

Figure 2. a) Desired image trajectory for the experiments. b) First revolution of the spiral trajectory.

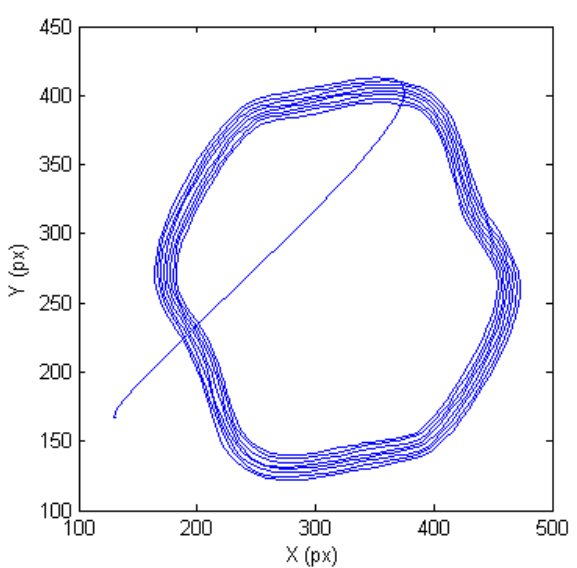

a)

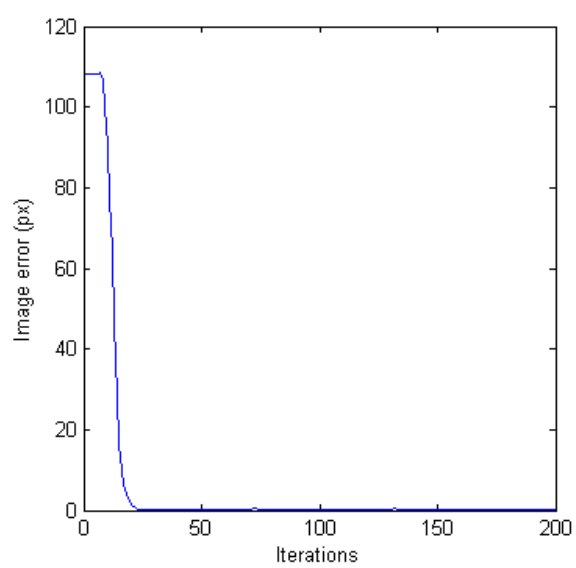

b)

Figure 3. Behavior in the image space without chaos control: a) Image trajectory. b) Image error module.
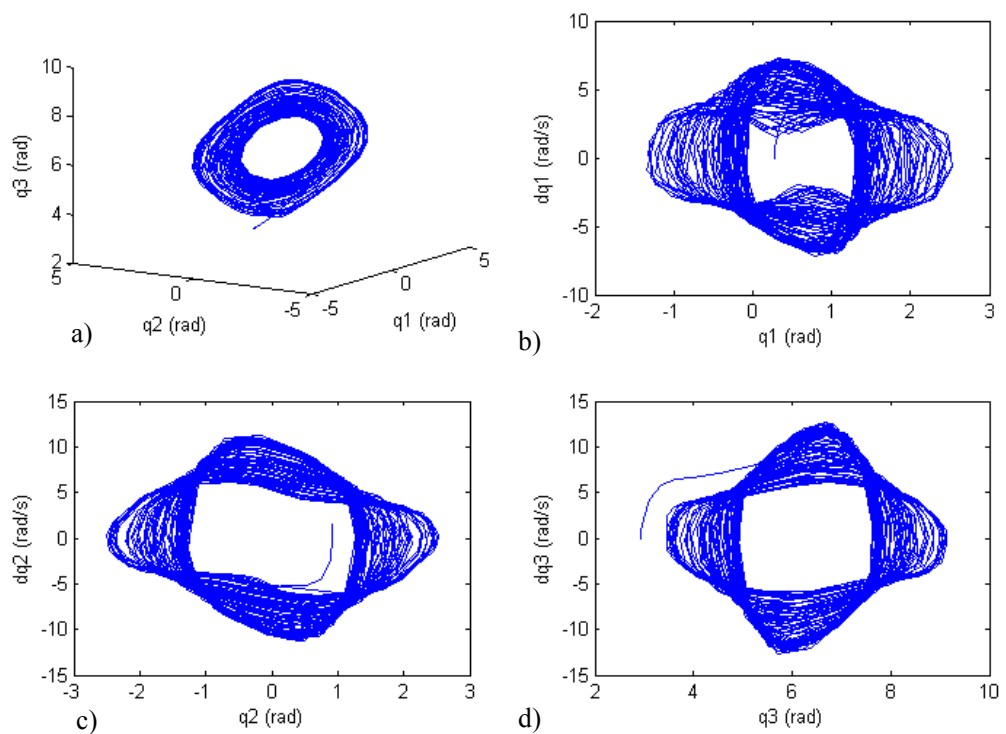

Figure 4. Phase portrait reconstructed from: a) $\mathrm{q}_{1}, \mathrm{q}_{2}, \mathrm{q}_{3}$. b) $\mathrm{q}_{1}, \dot{\mathrm{q}}_{1}$. c) $\mathrm{q}_{2}, \dot{\mathrm{q}}_{2}$. d) $\mathrm{q}_{3}, \dot{\mathrm{q}}_{3}$ without chaos control. 


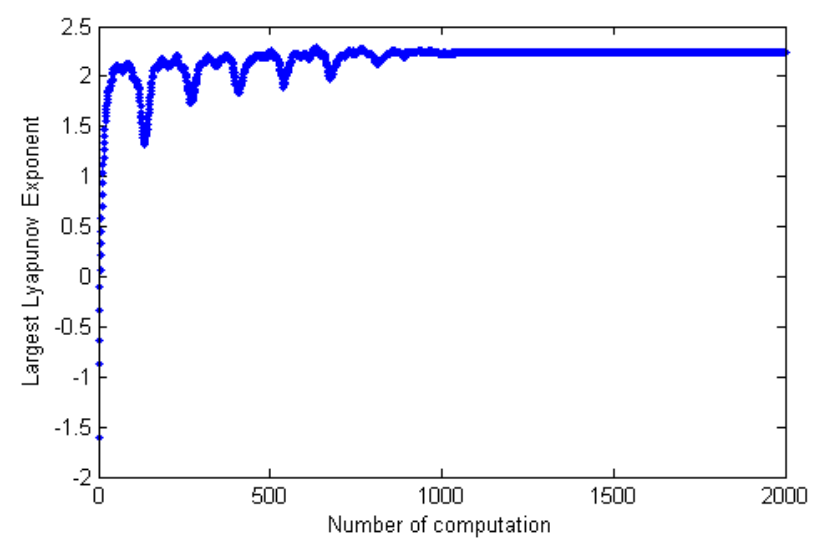

Figure 5. Largest Lyapunov Exponent diagram $(\operatorname{LLE}=2.238>0)$

In order to avoid chaotic behavior, the method proposed in [23] was applied initially, which performs an offline computation of the required gain for the DFC controller (this previous work does not consider the dynamical robot properties and do not employ visual information for the robot guidance). To do this, several simulations were carried out to determine the relationship between the gain $\mathrm{k}$ and the largest computed Lyapunov exponent of the system. This method supposes that a periodic joint motion is obtained using the gain value from which the largest Lyapunov exponent is negative. In practice, however, this method does not allow for complete compensation of the chaotic joint behavior when is applied in the direct visual servoing system proposed here. When simulated, the lowest value of the largest Lyapunov exponent is obtained when $\mathrm{k}=2.4$. Using this gain value in the real robot controller produces the phase portraits represented in Figure 6. This method does not allow for the elimination of the chaotic behavior due to several reasons. On one hand, the method uses simulations in order to determine the optimal gain value. Due to this, uncertainties in the robot dynamics are not taken into account, which is an important aspect to consider for machining processes, and the presence of measurement noise influences the system with unmodeled delays. Other hand, the tracked image trajectory is not perfectly periodic. Consequently, an adaptive method must be defined in order to optimally obtain the necessary gain value, and this method must update this value of $\mathrm{k}$ if it is necessary during the tracking. 

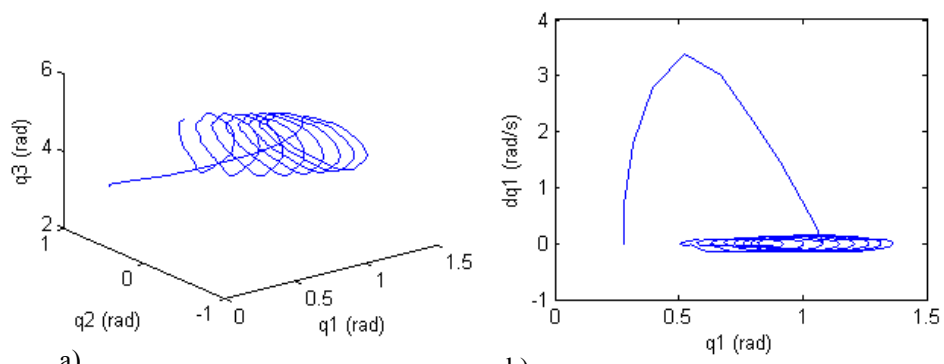

a)

b)
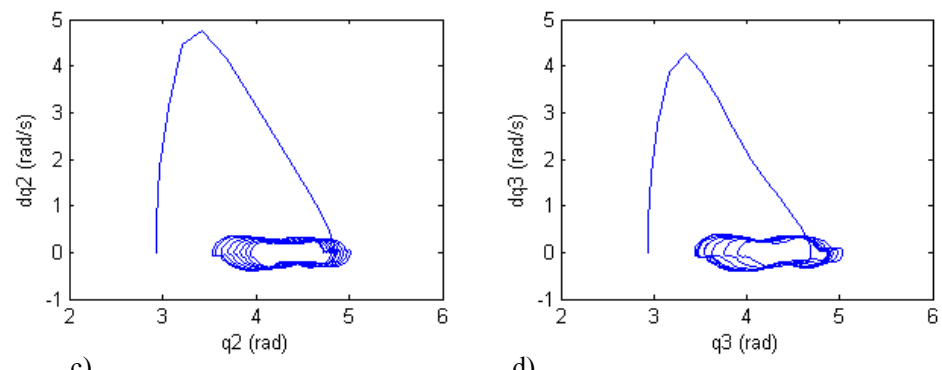

d)

Figure 6. Phase portrait reconstructed from: a) $\mathrm{q}_{1}, \mathrm{q}_{2}, \mathrm{q}_{3} \cdot$ b) $\mathrm{q}_{1}, \dot{\mathrm{q}}_{1} \cdot$ c) $\mathrm{q}_{2}, \dot{\mathrm{q}}_{2} \cdot$ d) $\mathrm{q}_{3}, \dot{\mathrm{q}}_{3} \cdot \mathrm{k}=2.4$.

The proposed adaptive method for determining the optimal value of $\mathrm{k}$ requires initializing the gain with a preliminary value $\left(\mathrm{k}_{1}\right)$. This initial value is equal to the gain value from which the minimum largest Lyapunov exponent is obtained in simulation. Additionally, the value of the delay time, $\varepsilon$, is fixed near the main period of the system, in this case, the delay $\varepsilon=2 \pi$ seconds. In order to take into account previous mentioned uncertainties and to adjust the gain k during tracking of the spiral image trajectory, the algorithm described in Figure 7 is applied. In this scheme, the variable $\beta$ is defined as the increment that will be applied to the gain in steps 6 and $7(\Delta \mathrm{k}=+\beta)$. At a given iteration $j, \Omega_{j} q_{i}$ is defined as the difference $\dot{q}_{i}\left(t_{j}-\varepsilon\right)-\dot{q}_{i}\left(t_{j}\right)$, where $t_{j}$ is the time at iteration $j$ and the index $\mathrm{i}$ indicates the joint under consideration (i=1,2,3). $\left|\Omega_{\mathrm{j}, \gamma} \mathrm{q}_{\mathrm{i}}\right|$ is the average value of the terms $\Omega_{\mathrm{g}} \mathrm{q}_{\mathrm{i}}$ where $\mathrm{g}$ $=\mathrm{j} \ldots \mathrm{j}+\gamma \cdot \gamma$ is an adjustable parameter. $\left|\Omega_{\mathrm{j}, \gamma}\right|$ is then computed from the average of the values of $\left|\Omega_{\mathrm{j}, \gamma} \mathrm{q}_{1}\right|,\left|\Omega_{\mathrm{j}, \gamma} \mathrm{q}_{2}\right|$, and $\left|\Omega_{\mathrm{j}, \gamma} \mathrm{q}_{3}\right|$. This value is saved as $|\Omega|_{\mathrm{B}}$. (step 3). In step 4 an increment is applied to $\mathrm{k}: \mathrm{k}=\mathrm{k}+\Delta \mathrm{k}$. Furthermore: j $=\mathrm{j}+\gamma$. In step 5 , using the new $\mathrm{k}$ obtained in step 4 , the value of $\left|\Omega_{\mathrm{j}, \gamma}\right|$ is computed (during $\gamma$ iterations). This value is stored as $|\Omega|_{\mathrm{E}}$. The loop represented in steps 4-9 continues until the complete trajectory is tracked. 


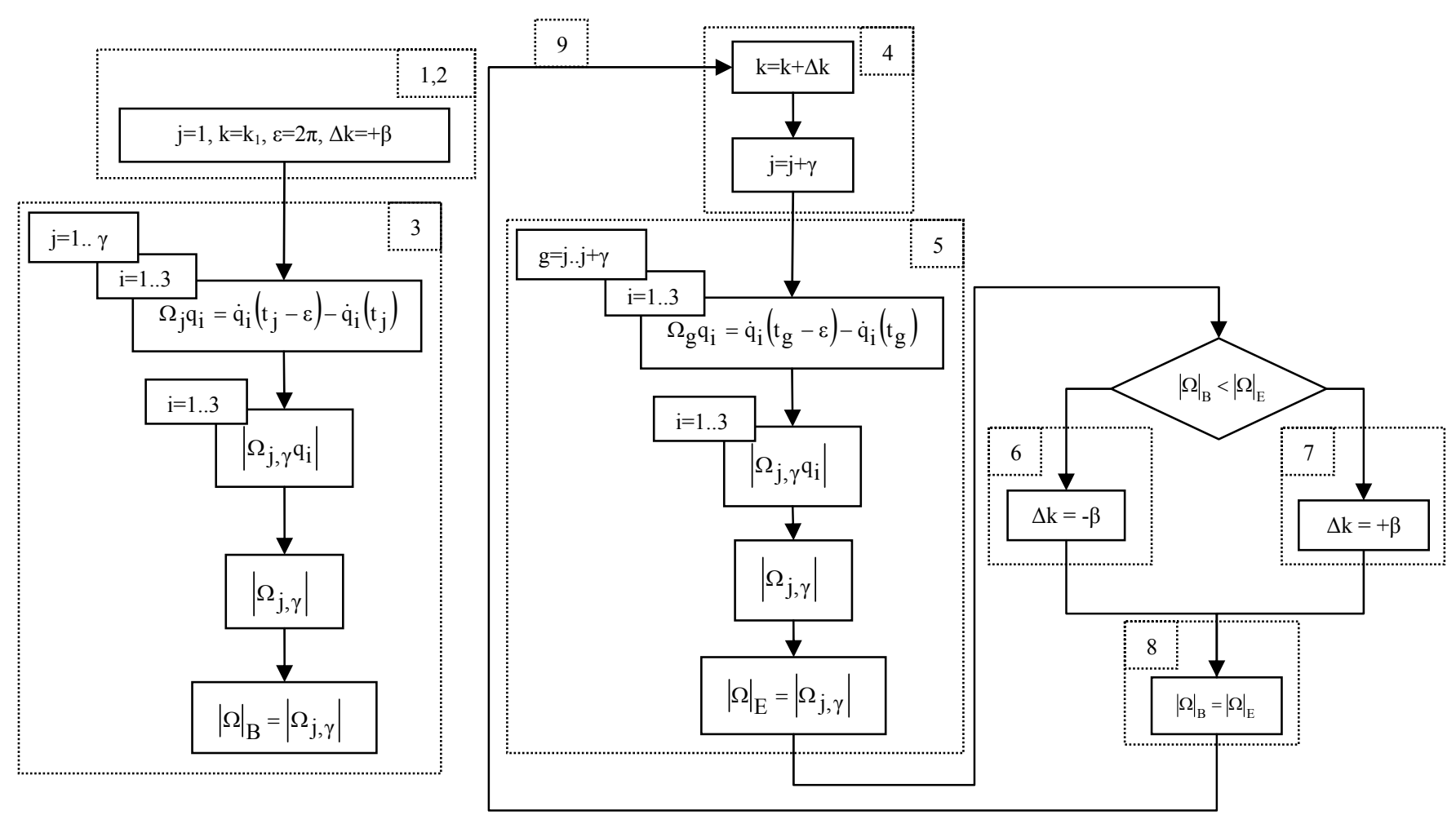

Figure 7. Adaptive method for determining the optimal value of $\mathrm{k}$.

The parameters $\beta$ and $\gamma$ are empirically adjusted to 0.1 and 10, respectively. A good estimation of the first gain value, $k_{1}$, is required in order to guarantee system's convergence. In the previously described algorithm, the value of $k_{1}$ is determined using offline simulations. As stated, this method does not guarantee optimal initialization of the gain. In order to perform this initialization using experimental values, an initial phase must be included in step 1 of the previous algorithm. This phase consists of tracking the first revolution of the spiral (Figure 2.b) until an acceptable initialization is achieved. Then, above described algorithm is applied during tracking of the image trajectory (represented in Figure 2.b) until the value of $\mathrm{k}$ is stabilized. In that moment begins the tracking of the spiral trajectory (Figure 2.a) which also applies the previous algorithm.

In the next paragraphs, the adaptive method previously described is applied to the tracking of the spiral trajectory in the image space. Figure 8 represents on the $\mathrm{x}$-axis a sampling of the gain values, $\mathrm{k}$, which were obtained during tracking. The corresponding values of $\left|\Omega_{\mathrm{j}, \gamma} \mathrm{q}_{1}\right|,\left|\Omega_{\mathrm{j}, \gamma} \mathrm{q}_{2}\right|,\left|\Omega_{\mathrm{j}, \gamma} \mathrm{q}_{3}\right|$ and $\left|\Omega_{\mathrm{j}, \gamma}\right|$ are represented on the $\mathrm{y}$-axis (the sub-indexes $\mathrm{j}$ and $\gamma$ are omitted in the figure). In simulations, the offline initialization of $\mathrm{k}$ produces a gain of $2.4\left(k_{1}=2.4\right)$. During the initial phase, the value of $k$ reaches 4 . Once the initial phase ends, the tracking of the spiral trajectory begins. Smooth variations in the gain allow the DFC to be adapted for measuring noise and other uncertainties in the experiment. 


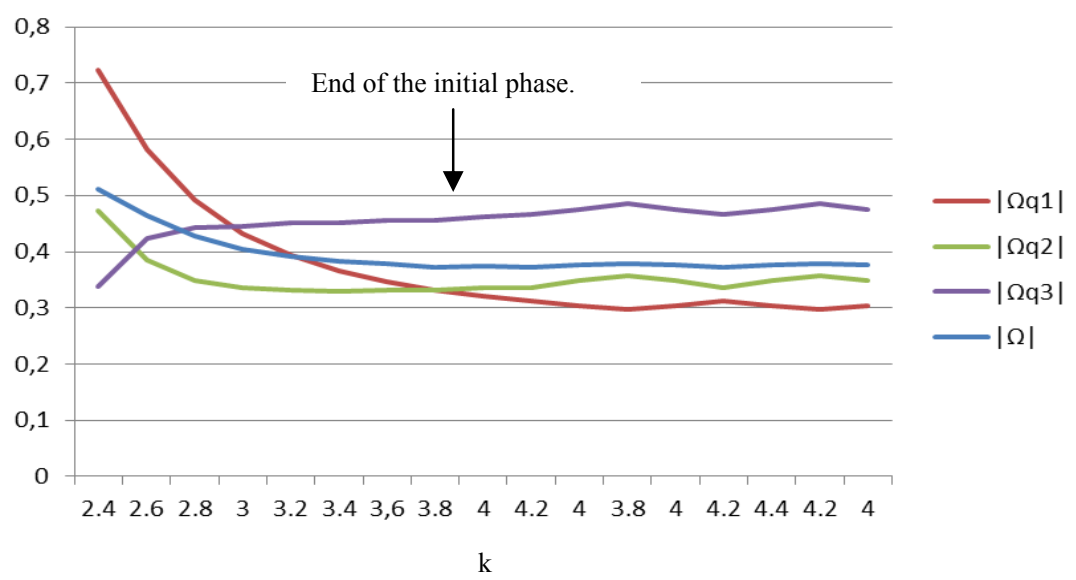

Figure 8. DFC gain values during the initial phase and tracking.

In order to demonstrate correct convergence of the adaptive method, the phase portraits obtained are shown in Figure 9. These phase portraits only represent the joint behavior during the tracking phase (the initial phase is omitted). The system begins with $\mathrm{k}=4$ and the gain value is adjusted during tracking to avoid chaotic behavior. The torques obtained without chaos control and those obtained using the adaptive method for chaos control, are compared in Figure 10. Additionally, the behavior in the image space with chaos control is indicated in Figure 11. As seen in Figure 10, the torques remain low once the initial phase ends (as previously indicated, the torques do not have to compensate the gravity force and only a smooth motion is required in the joint space). However, without chaos control the chaotic behavior requires high torques to perform the tracking of the spiral trajectory. Figure 11.a represents the trajectory obtained from the extracted visual feature in the image space. The tracking is correctly performed, and the image error remains low (see Figure 11.b). Finally, the trajectory described in the 3D space is represented in Figure 12. Figure 12.a illustrates the trajectory described by the robot during tracking of the four first cycles of the spiral trajectory. Practically, a repetitive motion is obtained in the joint space. A detail of the robot end during tracking is represented in Figure 12.b. A chaotic behavior is obtained when the adaptive method is not employed (see Figure 12.c). 

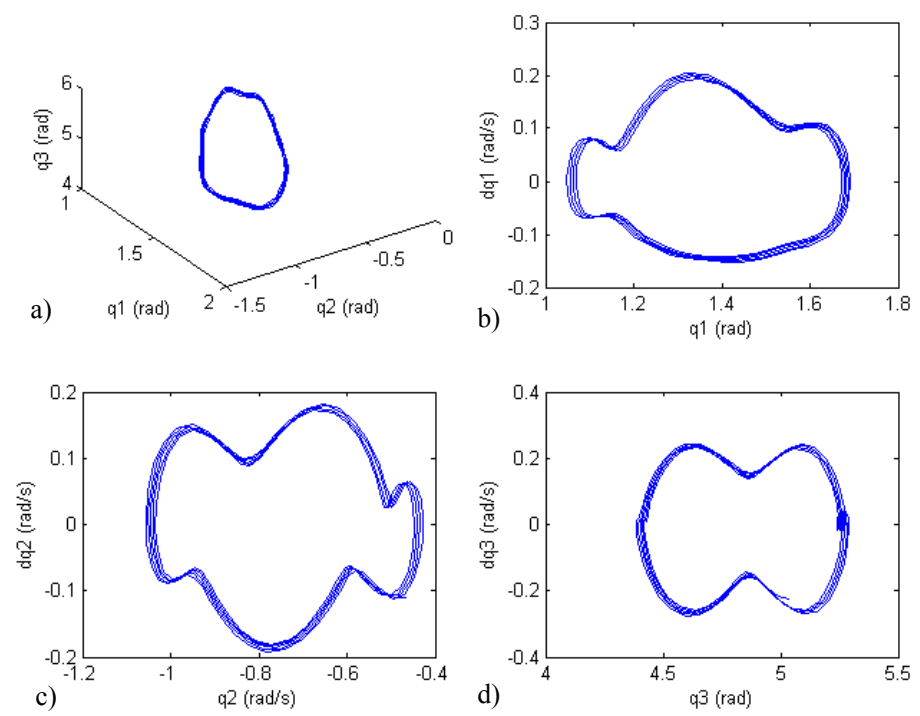

Figure 9. Phase portraits with chaos control (adaptive method) reconstructed from: a) $\left.\mathrm{q}_{1}, \mathrm{q}_{2}, \mathrm{q}_{3} . \mathrm{b}\right) \mathrm{q}_{1}, \dot{\mathrm{q}}_{1}$. c) $\mathrm{q}_{2}, \dot{\mathrm{q}}_{2}$. d) $\mathrm{q}_{3}, \dot{\mathrm{q}}_{3}$
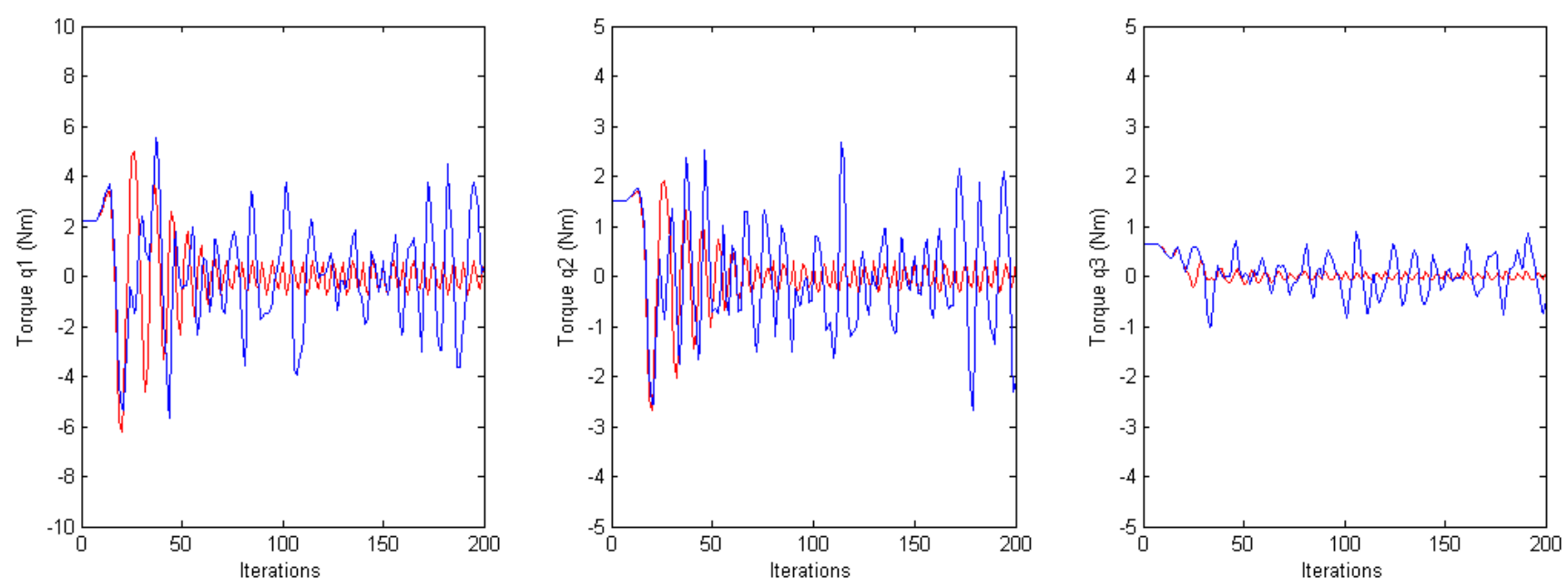

Figure 10. Torques during tracking with chaos control (red) and without chaos control (blue).

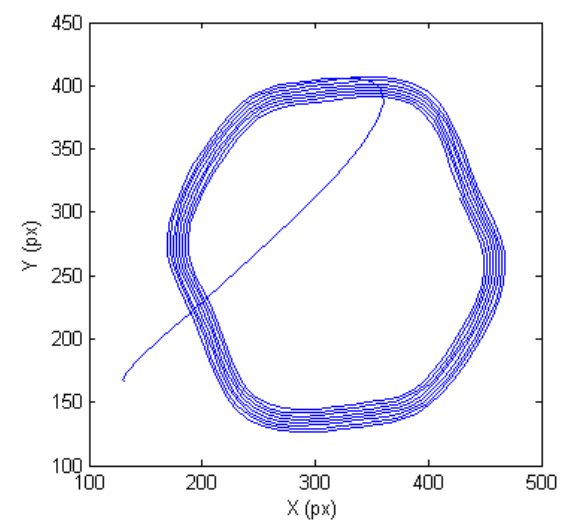

a)

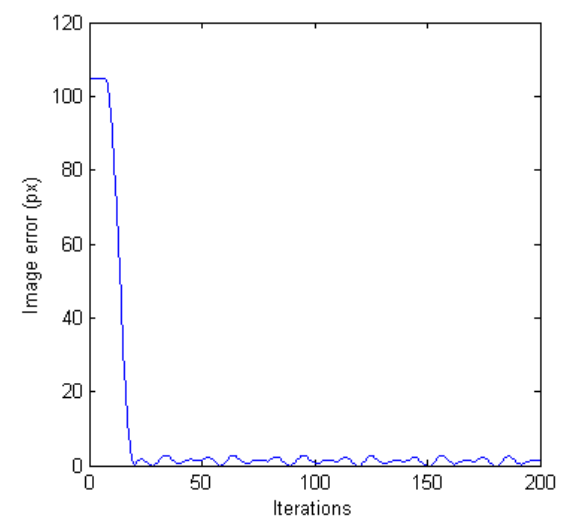

b)

Figure 11. Behavior in the image space with chaos control (adaptive method): a) Image trajectory. b) Image error module. 


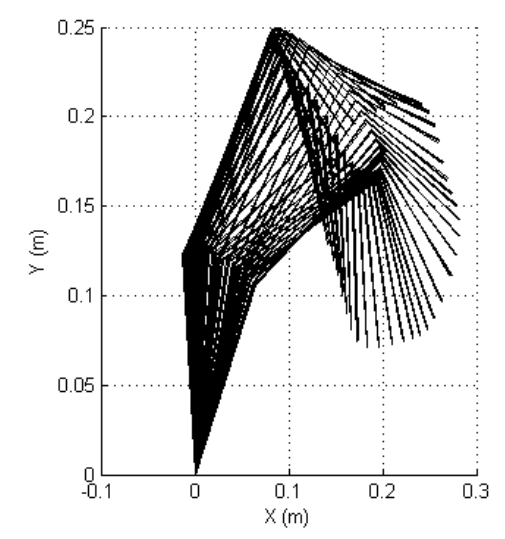

a)

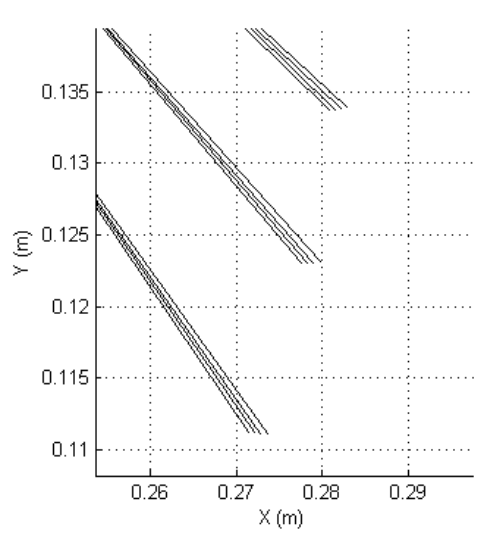

b)

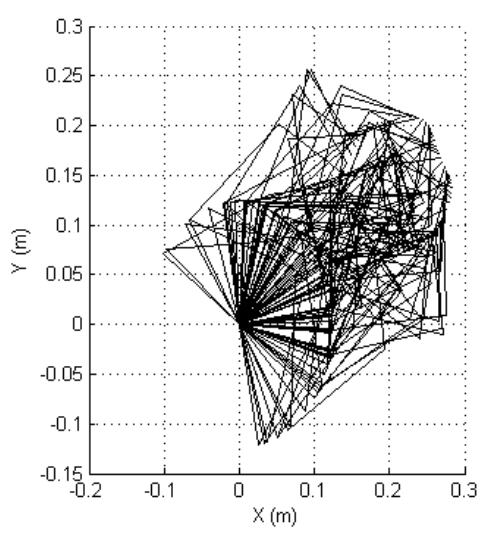

c)

Figure 12. Robot configurations during tracking: a) With chaos control (adaptive method). b) Detail of the robot-end during the tracking. c) Without chaos control.

\section{Evaluation of the proposed controller with different velocities}

The different experiments described in the previous section have shown the correct convergence of the direct visual controller proposed at Section 3. Moreover, the adaptive method presented on Section 4 allows the RT to perform the path tracking while avoiding chaotic joint behavior. These previous experiments all employ the same desired tracking velocity, which is defined by the parameter $\omega(\omega=0.5)$. In this section, however, the tracking behavior is analyzed when different velocities are applied in the machining process. To perform this analysis, the trajectory defined in equation (15) is tracked while using the proposed controller with chaos compensation. Figure 13 presents three experiments where the proportional and derivative matrices employed by the controller are $\mathbf{K}_{\mathrm{P}}=$ $\operatorname{diag}(10,10)$ and $\mathbf{K}_{\mathrm{D}}=\operatorname{diag}(50,50)$. The value of the parameter $\omega$ is 1,2 and $4 \mathrm{rad} / \mathrm{s}$ in the first, second and third experiments, respectively. As shown in Figure 13, the image error increases as the velocity increases. In contrast, a correct tracking is achieved at low and high velocities and the error remains low even when high velocities are required.

The joint torques obtained during the three experiments are presented in Figure 14. The torques obtained when chaos control is not applied are indicated in blue. In this particular case, the tracking is carried out correctly, but high torques are required for tracking. The torques obtained when the adaptive method for chaos control is used, are indicated in red. As shown in Figure 13, the chaos behavior is eliminated once the initial phase ends. From this moment, the torques remain low and a smooth evolution is obtained in the joint space. 

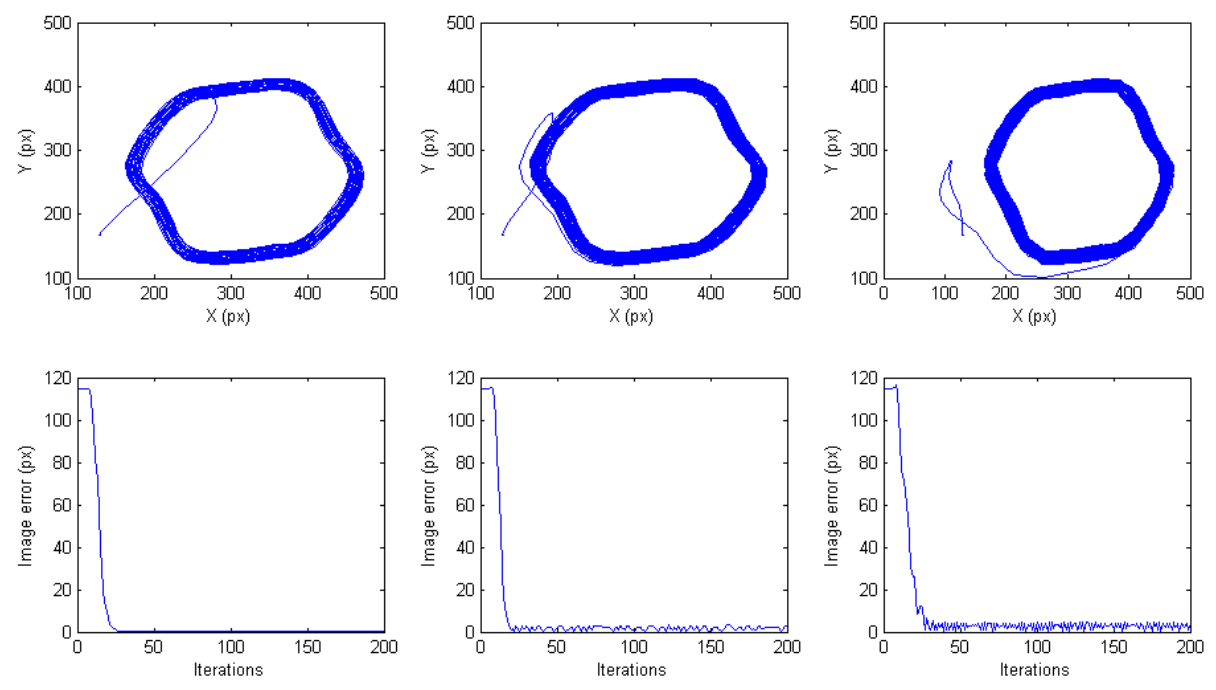

a)

b)

c)

Figure 13. Image trajectory and image error with chaos control: a) $\omega=1 \mathrm{rad} / \mathrm{s}, \mathrm{b}) \omega=2 \mathrm{rad} / \mathrm{s}, \mathrm{c}) \omega=4 \mathrm{rad} / \mathrm{s}$

a)

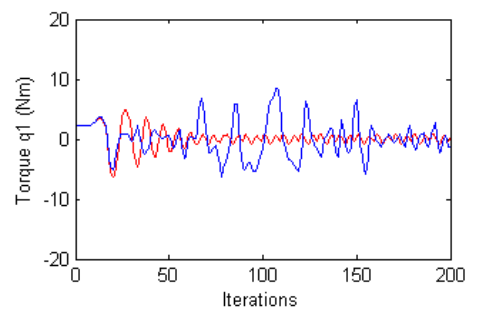

b)

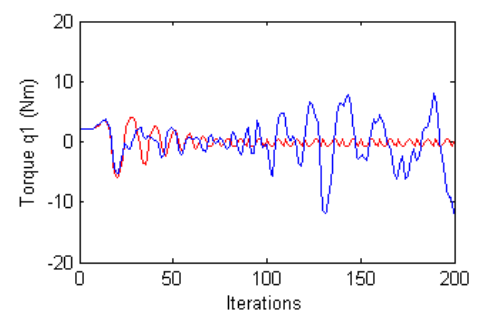

c)

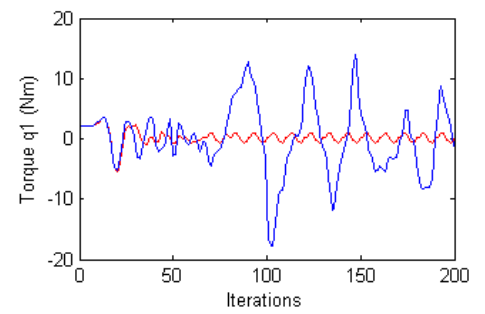

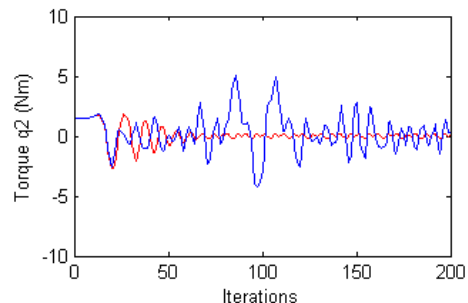
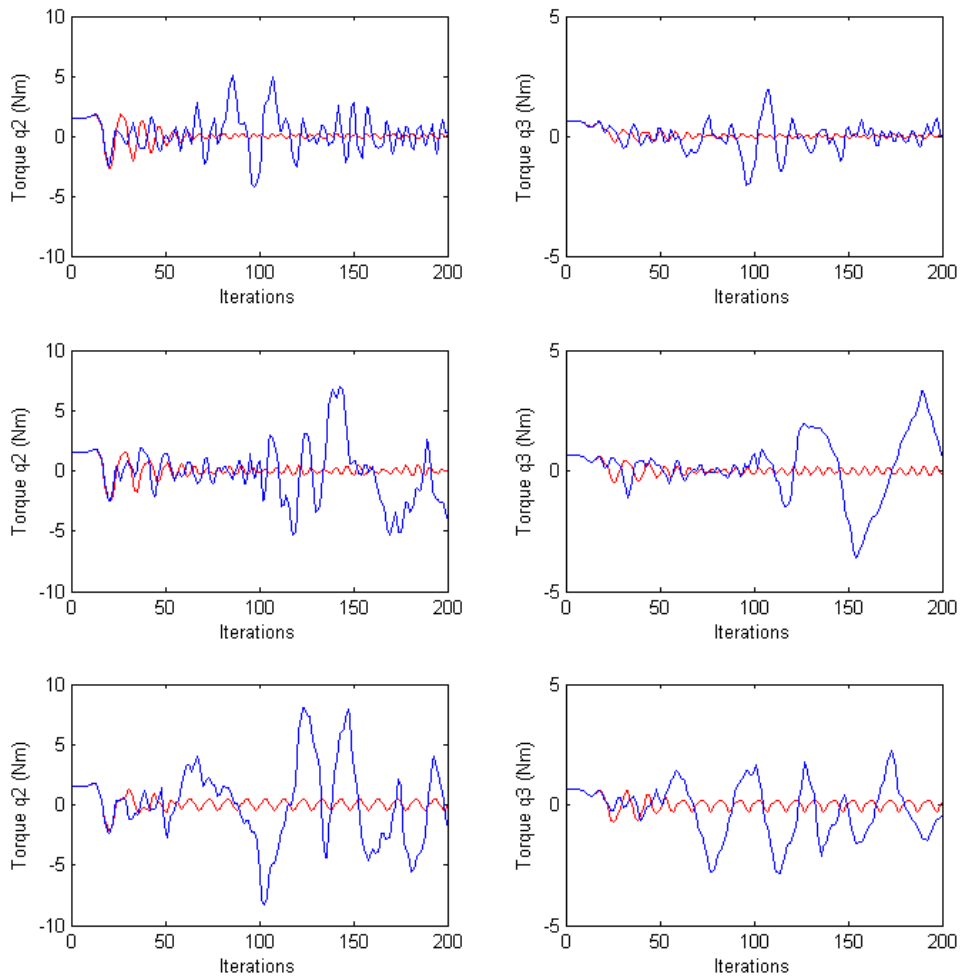

Figure 14. Torques during the tracking without chaos control (in blue) and with chaos control using the adaptive method (in red): a) $\omega=1 \mathrm{rad} / \mathrm{s}, \mathrm{b}) \omega=2 \mathrm{rad} / \mathrm{s}, \mathrm{c}) \omega=4 \mathrm{rad} / \mathrm{s}$.

\section{Conclusion}

This paper presents a 4 d.o.f. robot designed to be employed in non-contact machining task. A new direct visual servoing approach is presented in order to guide the RT by using visual information. The direct controller determines the torques to be applied to the RT motors in order to perform the tracking of a time-dependent image 
trajectory. The experiments show the correct behavior of the tracking method in low and high velocities.

In a milling process, the RT end-effector must track a repetitive or quasi-repetitive trajectory. In order to demonstrate the correct behavior of the proposed image controller performing these tasks, the tracking of a spiral trajectory is considered in the results section. A correct tracking is observed in this kind of image trajectories. Furthermore, as observed in the experimental results, a repetitive path tracked by the RT end-effector can produce a chaotic motion in the joint space. In order to avoid this problem, a chaos compensation method is proposed to obtain a repetitive joint behavior during tracking of repetitive or quasi-repetitive trajectories. This last aspect guarantees a smoother joint behavior and increases the machine safety obtaining predictable trajectories.

Currently, the authors are modifying the dynamic controller in order to take into account external forces existing in contact machining processes.

\section{Acknowledgements}

This work was funded by the Ministry of Science and Innovation of Spain Government through the research project DPI2011-22766 and DPI2012-32390.

\section{References}

[1] M.J. Tsai, J.F. Huang, W.L. Kao, Robotic polishing of precision molds with uniform material removal control, International Journal of Machine Tools \& Manufacture 49 (2009) 885-895.

[2] G. Vosniakos, E. Matsas, Improving feasibility of robotic milling through robot placement optimization, Robotics and Computer-Integrated Manufacturing 26 (5) (2010) 517-525.

[3] L. Andolfatto, S. Lavernhe, J.R.R. Mayer, Evaluation of servo, geometric and dynamic error sources on five-axis highspeed machine tool, International Journal of Machine Tools \& Manufacture 51 (2011) 787-796.

[4] W. Song, G. Wang, J. Xiao, G. Wang, Y. Hong, Research on multi-robot open architecture of an intelligent CNC system based on parameter-driven technology, Robotics and Computer-Integrated Manufacturing 28 (2012) 326-333.

[5] P. Sungsik, K. Sun-Ho, C. Hyunbo, Kernel software for efficiently building, re-configuring, and distributing an open CNC controller, International Journal of Advanced Manufacturing Technology 27 (2006) 788-796.

[6] S. Mekid, P. Pruschek, J. Hernandez, Beyond intelligent manufacturing: a new generation of flexible intelligent NC machines, Mechanism and Machine Theory 44(2) (2009) 466-476.

[7] H. Son, H. Choi, H. W. Park, Design and dynamic analysis of an arch-type desktop reconfigurable machine, International Journal of Advanced Manufacturing Technology 50 (2010) 575-584.

[8] G. Al-Kindi, H. Zughaer, An Approach to Improved CNC Machining Using Vision-Based System, Materials and Manufacturing Processes, vol. 27, pp. 765-774, 2012.

[9] G. Al-Kindi, R. Baul, K. Gill, Vision-controlled CNC machines, Computing \& Control Engineering Journal, vol. 4, pp. 92-96, 1993.

[10] A. Eladawi, E. Gadelmawla, I. Elewa, A. Abdel-Shafy, An application of computer vision for programming computer numerical control machines, Proceedings of the Institution of Mechanical Engineers Part B-Journal of Engineering Manufacture, vol. 217, pp. 1315-1324, 2003.

[11]T. Cano, F. Chapelle, J.-M. Lavest, P. Ray, A new approach to identifying the elastic behaviour of a manufacturing machine, International Journal of Machine Tools and Manufacture 48(14) (2008) 1569-1577.

[12] C. Hong, S. Ibaraki, C. Oyama, Graphical presentation of error motions of rotary axes on a five-axis machine tool by static R-test with separating the influence of squareness errors of linear axes, International Journal of Machine Tools and Manufacture 59 (2012) 24-33. 
[13]C. Hu, B. Yao, Q.Wang, Z. Chen, C. Li, Experimental investigation on high-performance coordinated motion control of high-speed biaxial systems for contouring tasks, International Journal of Machine Tools and Manufacture 51(9) (2011) 677-686.

[14]F. Chaumette, S. Hutchinson, Visual Servo Control, Part I: Basic Approaches, IEEE Robotics and Automation Magazine 13(4) (2006) 82-90.

[15] G. J. García, C. A. Jara, J. Pomares, F. Torres, F, Direct visual servo control of a robot to track trajectories in supervision tasks,. In Proceedings of IEEE ICARCV 1 (2010) 1434-1439.

[16] I. N. Tansel, C. Erkal, T. Keramidas, The chaotic characteristics of three dimensional cutting, International Journal of Machine Tools and Manufacture 32 (6) (1992) 811-827.

[17] G. Litak, Chaotic vibrations in a regenerative cutting process, Chaos, Solitons \& Fractals 13 (2002) 1531-1535.

[18] J. Pomares, I. Perea, G. J. García, C. A. Jara, J. A. Corrales, F. Torres, A Multi-Sensorial Hybrid Control for Robotic Manipulation in Human-Robot Workspaces, Sensors 11(10) (2011) 9839-9862.

[19] E. Malis, P. Rives, Robustness of image-based visual servoing with respect to depth distribution errors, In Proceedings of the IEEE International Conference on Robotics and Automation (2003) 1056-1061.

[20]Z. Zhang, Three-dimensional reconstruction under varying constraints on camera geometry for robotic navigation scenarios, Thesis, University of Massachusetts Amherst, 1996.

[21] T. Yoshikawa, Manipulability of Robotic Mechanisms, International Journal of Robotics Research 4(2) (1985) 3-9 1985.

[22] K. Pyragas, Continuous control of chaos by self-controlling feedback, Physics Letters, 170 (1992) 421-428.

[23]Z. Liu, L. Li, H. Zhang, Control Chaos in a spatially redundant manipulator with flexible link, in Proceedings of 12th World Congress in Mechanism and Machine Science (2007). 


\section{Figure and Table captions}

Figure 1. Robot tool.

Figure 2. a) Desired image trajectory for the experiments. b) First revolution of the spiral trajectory.

Figure 3. Behavior in the image space without chaos control: a) Image trajectory. $b$ ) Image error module.

Figure 4. Phase portrait reconstructed from: a) $\mathrm{q}_{1}, \mathrm{q}_{2}, \mathrm{q}_{3}$. b) $\mathrm{q}_{1}, \dot{\mathrm{q}}_{1}$. c) $\mathrm{q}_{2}, \dot{\mathrm{q}}_{2}$. d) $\mathrm{q}_{3}, \dot{\mathrm{q}}_{3}$ without chaos control.

Figure 5. Largest Lyapunov Exponent diagram $(\operatorname{LLE}=2.238>0)$

Figure 6. Phase portrait reconstructed from: a) $\mathrm{q}_{1}, \mathrm{q}_{2}, \mathrm{q}_{3}$. b) $\left.\left.\mathrm{q}_{1}, \dot{\mathrm{q}}_{1} \cdot \mathrm{c}\right) \mathrm{q}_{2}, \dot{\mathrm{q}}_{2} \cdot \mathrm{d}\right) \mathrm{q}_{3}, \dot{\mathrm{q}}_{3} \cdot \mathrm{k}=2.4$.

Figure 7. Adaptive method for determining the optimal value of $\mathrm{k}$.

Figure 8. DFC gain values during the initial phase and tracking.

Figure 9. Phase portraits with chaos control (adaptive method) reconstructed from: a) $\mathrm{q}_{1}, \mathrm{q}_{2}, \mathrm{q}_{3}$. b) $\mathrm{q}_{1}, \dot{\mathrm{q}}_{1}$. c) $\mathrm{q}_{2}, \dot{\mathrm{q}}_{2}$.

d) $\mathrm{q}_{3}, \dot{\mathrm{q}}_{3}$.

Figure 10. Torques during tracking with chaos control (red) and without chaos control (blue).

Figure 11. Behavior in the image space with chaos control (adaptive method): a) Image trajectory. b) Image error module.

Figure 12. Robot configurations during tracking: a) With chaos control (adaptive method). b) Detail of the robotend during the tracking. c) Without chaos control.

Figure 13. Image trajectory and image error with chaos control: a) $\omega=1 \mathrm{rad} / \mathrm{s}, \mathrm{b}) \omega=2 \mathrm{rad} / \mathrm{s}, \mathrm{c}) \omega=4 \mathrm{rad} / \mathrm{s}$.

Figure 14. Torques during the tracking without chaos control (in blue) and with chaos control using the adaptive method (in red): a) $\omega=1 \mathrm{rad} / \mathrm{s}, \mathrm{b}) \omega=2 \mathrm{rad} / \mathrm{s}, \mathrm{c}$ ) $\omega=4 \mathrm{rad} / \mathrm{s}$.

Table 1: Motor parameters of the joints $\mathrm{q}_{0}, \mathrm{q}_{1}, \mathrm{q}_{2}, \mathrm{q}_{3}$.

Table 2: Gear parameters of the joints $\mathrm{q}_{0}, \mathrm{q}_{1}, \mathrm{q}_{2}, \mathrm{q}_{3}$. 